\title{
Angular dependence of the magnetoelectric effect in orthorhombic $\mathrm{HoMnO}_{3}$ films
}

\author{
M. Ziese, A. Setzer, R. Wunderlich, C. Zandalazini, and P. Esquinazi \\ Division of Superconductivity and Magnetism, University of Leipzig, D-04103 Leipzig, Germany \\ (Received 2 December 2010; revised manuscript received 16 September 2011; published 19 December 2011)
}

\begin{abstract}
Epitaxial orthorhombic $\mathrm{HoMnO}_{3}$ films were grown on $\mathrm{Nb}$-doped $\mathrm{SrTiO}_{3}(001)$ single-crystal substrates. X-ray diffractometry showed a uniform crystallographic orientation with the $c$ axis along the substrate normal and an anisotropic compressive stress along the $b$ axis of the Pbnm structure. The magnetization of the films was dominated by the paramagnetism of the $\mathrm{Ho}^{3+}$ ions; the latter showed a strong anisotropy with respect to the in-plane and perpendicular-to-plane magnetic field direction. The rotational anisotropy of the magnetoelectric effect was measured for magnetic field rotation in the $(110)_{o},(1 \overline{1} 0)_{o}$, and $(001)_{o}$ planes. Whereas magnetic field rotation in the $(110)_{o}$ and $(1 \overline{1} 0)_{o}$ planes showed a twofold pattern with the smallest magnetoelectric effect observed in magnetic fields along the $c$ axis, in-plane $(001)_{o}$ magnetic field rotations revealed an intricate rotational symmetry. A magnetic-field-induced crossover was observed from a low-field region with fourfold rotation patterns to a high-field region with rotation patterns up to the 12 th order. This complex rotational symmetry arises from spin-orbit coupling of the $\mathrm{Ho}^{3+}$ moments that induces a modulation of the magnetoconductance as well as a magnetoelectric effect through the Maxwell-Wagner mechanism.
\end{abstract}

DOI: 10.1103/PhysRevB.84.214424

PACS number(s): 75.85.+t, 77.55.Nv, 75.47.Lx

\section{INTRODUCTION}

Hexagonal and orthorhombic manganites have attracted tremendous research interest, since these systems often show a strong coupling between electric and magnetic degrees of freedom. ${ }^{1-4}$ Of interest for the growth of heterostructures and superlattices are the orthorhombic phases, ${ }^{5-9}$ since these can be combined with other ferromagnetic and ferroelectric perovskites such as $\mathrm{SrRuO}_{3}, \mathrm{La}_{0.7} \mathrm{Sr}_{0.3} \mathrm{MnO}_{3}$, and $\mathrm{BaTiO}_{3}$. Orthorhombic $\mathrm{HoMnO}_{3}$ crystallizes in the $\mathrm{GdFeO}_{3}$-type structure $(\mathrm{Pbnm}) .{ }^{10}$ Below $41 \mathrm{~K}$ the $\mathrm{Mn}^{3+}$ ions order in an incommensurate spin-density wave; below $26 \mathrm{~K}$ this magnetic structure transforms into an antiferromagnetic $E$-type structure. ${ }^{10,11}$ The latter magnetic structure breaks inversion symmetry and leads to the formation of an improper ferroelectric state. ${ }^{12-15}$ Further the Ho sublattice magnetization orders antiferromagnetically at about $9 \mathrm{~K} .{ }^{16}$ There are indications that the Ho moments stabilize the ionic displacements in the ferroelectric phase, ${ }^{17}$ but the main focus of research has been on the properties of the $\mathrm{MnO}_{2}$ sublattice so far.

Since there are no large single crystals of the orthorhombic $\mathrm{HoMnO}_{3}$ phase, ${ }^{17,18}$ studies of epitaxial o- $\mathrm{HoMnO}_{3}$ thin films might provide more information on magnetoelectric symmetries. In this work epitaxial orthorhombic $\mathrm{HoMnO}_{3}$ films were grown on $\mathrm{Nb}$-doped $\mathrm{SrTiO}_{3}$ single-crystal substrates. The rotational symmetry of the dielectric permittivity of the films was studied for magnetic field rotation in various film planes and was found to be intricately controlled by the magnetic field strength.

\section{EXPERIMENT}

Orthorhombic $\mathrm{HoMnO}_{3}$ films were grown by pulsed laser deposition from a stoichiometric polycrystalline target onto $0.5 \% \mathrm{Nb}$-doped $\mathrm{SrTiO}_{3}$ (001) substrates. An excimer laser (Lambda Physik) operating at a wavelength of $248 \mathrm{~nm}(\mathrm{KrF})$, a repetition rate of $10 \mathrm{~Hz}$, and a fluence of about $1.5 \mathrm{~J} / \mathrm{cm}^{2}$ was used for the ablation. Oxygen partial pressure during deposition was $0.1 \mathrm{mbar}$ and substrate temperature was $800^{\circ} \mathrm{C}$; see also Ref. 19 for further details. The thickness of the films was estimated from the deposition time to be $d \simeq$ $200 \mathrm{~nm}$ with an uncertainty of about $10 \%$. Three o- $\mathrm{HoMnO}_{3}$ films were chosen for further study which were used for magnetotransport, magnetometry, and x-ray diffractometry measurements, respectively.

Structural characterization of the films was made by $\mathrm{x}$ ray diffraction (XRD) with a Philips X'pert system using $\mathrm{Cu} \mathrm{K}_{\alpha 1}$ radiation. Magnetic characterization of the films was carried out by SQUID magnetometry (Quantum Design model MPMS-7). The magnetocapacitance and -conductance were measured in an Oxford Instruments cryostat using an AndeenHagerling capacitance bridge (model AH 2500A) operating at $1 \mathrm{kHz}$ and an ac voltage of $100 \mathrm{mV}$. Assuming a parallel circuit between capacitance $C$ and resistance $R=G^{-1}$, where $G$ denotes the conductance, the Andeen-Hagerling bridge measures the inverse complex impedance $Z^{-1}=i \omega C+G$. Here the complex capacitance $\hat{C}=(i \omega Z)^{-1} \equiv C^{\prime}-i C^{\prime \prime}=C-i G / \omega$ is reported. The top contact was made by evaporating a gold square of $A=0.0625 \mathrm{~mm}^{2}$ area onto the $\mathrm{o}-\mathrm{HoMnO}_{3}$ film; the conducting $\mathrm{Nb}: \mathrm{SrTiO}_{3}$ substrate was used as the bottom contact. Both top and bottom contact were contacted with gold wires and silver paint. The dielectric permittivity was calculated from the capacitance using $\hat{\epsilon}=\epsilon^{\prime}-i \epsilon^{\prime \prime}=\hat{C} / C_{0}$, where $C_{0}=\epsilon_{0} A / d$ and $\epsilon_{0}$ denotes the vacuum permittivity. The sample holder could be rotated with respect to the magnetic field with a relative resolution of about $0.01^{\circ}$. The field direction was defined relative to the edges of the quadratic $\mathrm{Nb}: \mathrm{SrTiO}_{3}$ substrate (in-plane $[100]_{c}$ and $[010]_{c}$ directions) with an accuracy of about $\pm 3^{\circ}$.

\section{RESULTS}

\section{A. Structural characterization}

Orthorhombic $\mathrm{HoMnO}_{3}$ crystallizes in the Pbnm crystal structure with bulk lattice constants $a=0.527 \mathrm{~nm}, b=$ $0.584 \mathrm{~nm}$, and $c=0.736 \mathrm{~nm} .{ }^{10} \mathrm{SrTiO}_{3}$ crystallizes in the $P m 3 m$ structure with a lattice constant $a_{S T O}=0.3905 \mathrm{~nm}$. 


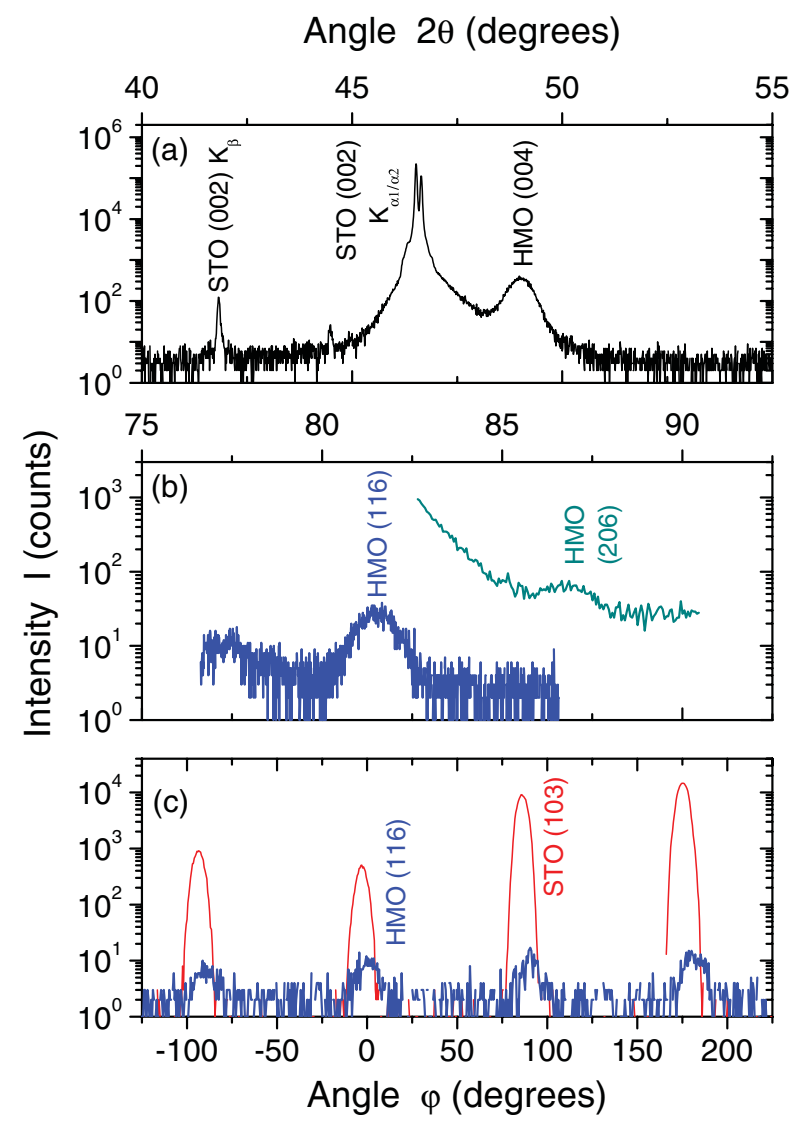

FIG. 1. (Color online) $\theta-2 \theta$ scans of the o- $-\mathrm{HoMnO}_{3}$ (a) (004) and (b) (116) and (206) reflections. (c) $\varphi$ scan of the o- $-\mathrm{HoMnO}_{3}$ (116) reflections.

The lattice mismatch of $a / \sqrt{2}, b / \sqrt{2}$, and $c / 2$ with the $\mathrm{SrTiO}_{3}$ lattice constant is rather large with $-4.6 \%,+5.7 \%$, and $-5.8 \%$, respectively. In general orthorhombic perovskites have two epitaxial relations with cubic $\mathrm{SrTiO}_{3}$ and might grow either with the $[001]_{o}$ or the $[110]_{o}$ direction along the substrate normal; see, e.g., $\mathrm{SrRuO}_{3}$, which grows (110)o oriented. ${ }^{20}$ Since the difference between $a$ - and $b$-lattice constants is substantial, one might further expect twinning or more complex pattern formation as was indeed found for $\mathrm{TbMnO}_{3}$ films grown on $\mathrm{SrTiO}_{3}(001){ }^{21}$

In Fig. $1 \theta-2 \theta$ and $\varphi$ scans of an $\mathrm{o}-\mathrm{HoMnO}_{3}$ film are presented. Figure 1(a) shows a conventional $\theta-2 \theta$ scan, with the angle range around the $\mathrm{SrTiO}_{3}(002)$ reflection enlarged. This scan showed only the presence of $(00 l) \mathrm{SrTiO}_{3}$ reflections accompanied by film reflections at somewhat larger angles. The latter reflections were indexed as $(002 l)$ o- $\mathrm{HoMnO}_{3}$ reflections yielding a $c$-axis parameter $c=0.7430 \pm 0.0005 \mathrm{~nm}$. This is slightly larger than the bulk value, indicating the presence of compressive stress exerted by the substrate on the film. Figure 1(b) shows $\theta-2 \theta$ scans of the o- $\mathrm{HoMnO}_{3}(116)$ and (206) reflections at $81.65^{\circ}$ and at $86.9^{\circ}$. From this the in-plane lattice constants were calculated as $a=0.526 \pm 0.002 \mathrm{~nm}$ and $b=0.569 \pm 0.002 \mathrm{~nm}$. Figure 1 (c) shows $\varphi$ scans of the $\mathrm{SrTiO}_{3}$ (103) and o- $\mathrm{HoMnO}_{3}$ (116) reflections; both scans showed four peaks consistent with cubic and orthorhombic symmetry.
This analysis leads to the following conclusions: (i) The o- $\mathrm{HoMnO}_{3}$ films grow $c$-axis oriented; (ii) the films show to a large extent a uniform orientation of the in-plane $a$ and $b$ axes; (iii) whereas the $a$-axis lattice constant of the films is equal to the bulk value, the $b$-axis parameter is $2.5 \%$ smaller than that of the bulk; i.e., in the $b$ direction the films are under compressive stress. The unit cell volume of the films is $-1.8 \%$ smaller than the bulk value.

In oxide films strain relaxation of the lattice parameters in general sets in for film thicknesses below $100 \mathrm{~nm}^{22-24}$ The exact value of the critical thickness depends on the lattice mismatch between film and substrate and on the growth mode. The $\mathrm{x}$-ray data indicate that the films grew partially strain relaxed and that the strain relaxation is anisotropic with the $a$-axis lattice constant being fully strain relaxed, whereas there is still compressive strain along the $b$ direction. The latter observation was also made for $\mathrm{TbMnO}_{3}$ films. ${ }^{25}$ Anisotropic strain relaxation is not unusual in an orthorhombic structure, the less so since Poisson's ratio might even change sign along particular lattice directions. ${ }^{26}$ The films will certainly have growth defects to accommodate the strain relaxation. However, and this is the most important conclusion from the $\mathrm{x}$-ray analysis, the films show a uniform orientation of the $a$ and $b$ axes; i.e., twinning does not occur in large areas. Therefore the films can be used for an extensive analysis of the angle-dependent magnetoelectric effect.

\section{B. Magnetic properties}

Neutron diffractometry has revealed three magnetic transitions in orthorhombic $\mathrm{HoMnO}_{3}:{ }^{10,11}$ At $41 \mathrm{~K}$ the $\mathrm{Mn}^{3+}$ ions order in an incommensurate spin-density wave with the magnetic moments in the (100) plane and modulated by a wave vector along the $a$ axis. At $26 \mathrm{~K}$ this magnetic structure locks into the commensurate AFM $E$-type magnetic structure with $\mathrm{Mn}^{3+}$ spins parallel to $b$ forming zigzag chains in the $a-b$ plane along the $a$ axis. This Mn order is stable to the lowest temperatures measured in that study $(1.4 \mathrm{~K})$; the transition temperatures are independent of magnetic field. Spiral magnetic order of the $\mathrm{Ho}^{3+}$ ions occurs at $6.5 \mathrm{~K}$ with the Ho moments lying in the (110) plane. The latter transition is easily suppressed by an applied magnetic field.

Figure 2(a) shows the zero-field-cooled (ZFC) and fieldcooled (FC) magnetic moment of an $\mathrm{o}-\mathrm{HoMnO}_{3}$ film for an in-plane $\left([110]_{o}\right.$ or $\left.[1 \overline{1} 0]_{o}\right)$ direction. The curves indicate paramagnetic behavior; no obvious phase transition or branching of the FC-ZFC curves at $41 \mathrm{~K}$ was observed. This result is in contrast to the results of Refs. $6,7,27$, but in agreement with Refs. $8,9,16$. The data of Wood et al. ${ }^{16}$ on polycrystalline orthorhombic manganites show that the antiferromagnetic transition at $41 \mathrm{~K}$ is masked by the strong paramagnetic $\mathrm{Ho}^{3+}$ contribution in $\mathrm{o}-\mathrm{HoMnO}_{3}$, whereas it can be clearly observed in o- $\mathrm{YMnO}_{3}$. The data presented here would be in agreement with this interpretation. There are some indications that the transition around $41 \mathrm{~K}$ observed in some samples by magnetization measurements might be at least partially related to hausmannite $\left(\mathrm{Mn}_{3} \mathrm{O}_{4}\right)$ precipitates, ${ }^{16,19,28}$ but this issue has not yet been settled. 


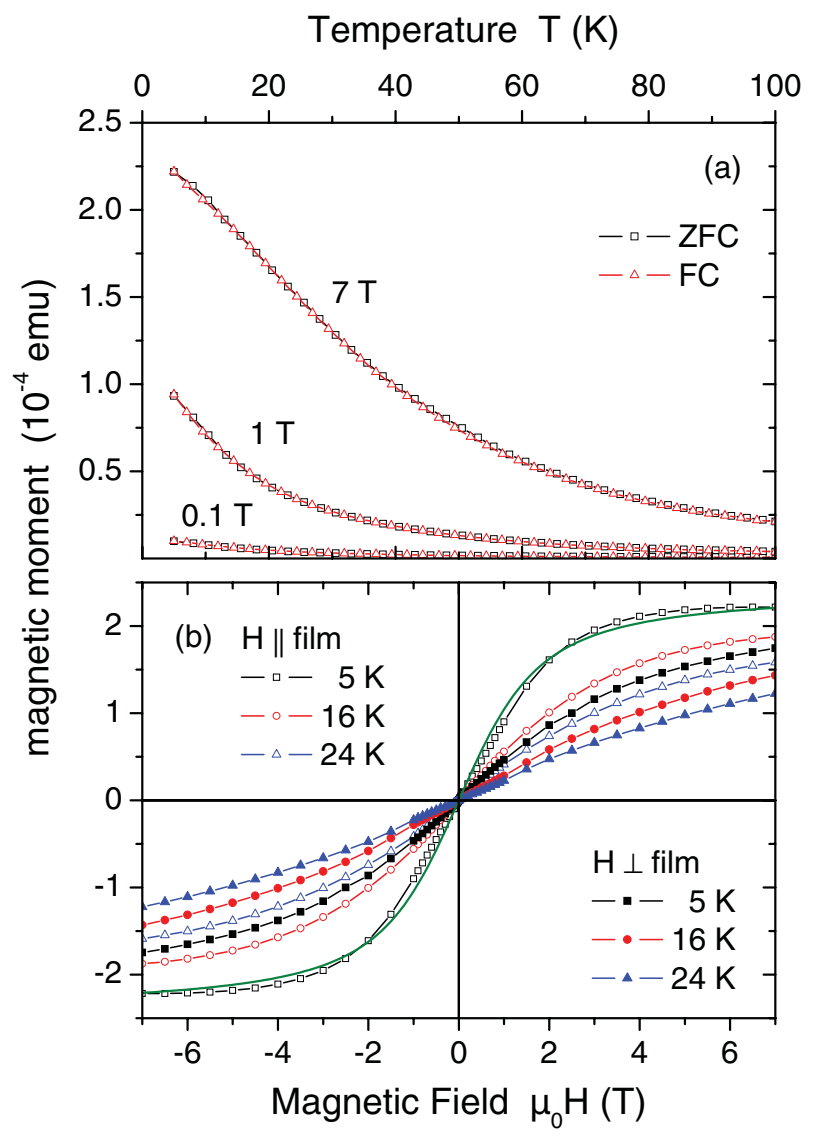

FIG. 2. (Color online) (a) Magnetic moment vs temperature along an in-plane direction $\left([110]_{o}\right.$ or $\left.[1 \overline{1} 0]_{o}\right)$ for applied fields of $0.1,1$, and 7 T. Both zero-field-cooled (ZFC) and field-cooled (FC) data are shown. (b) Hysteresis loops measured along an in-plane ([110]。 or $[1 \overline{1} 0]_{o}$, open symbols) and the perpendicular-to-plane direction ([001 $]_{o}$, solid symbols). The solid line in (b) was calculated with the Brillouin function using $J=8$ and $g=5 / 4$.

Figure 2(b) shows hysteresis curves measured at 5, 16, and $24 \mathrm{~K}$ both for an in-plane $\left([110]_{o}\right.$ or $\left.[1 \overline{1} 0]_{o}\right)$ and the perpendicular-to-plane $\left([001]_{o}\right)$ direction. The field dependence of the magnetic moment is dominated by the paramagnetic $\mathrm{Ho}^{3+}$ contribution. This is anisotropic with saturation achieved in high in-plane fields at $5 \mathrm{~K}$, but not in out-of-plane fields up to $7 \mathrm{~T}$. Therefore the magnetic hard axis of the $\mathrm{Ho}^{3+}$ spins is along the orthorhombic $c$ axis. A fit of the Brillouin function using the free ion $\mathrm{Ho}^{3+}$ values $S=2$, $L=6, J=8$, and $g=5 / 4$ yields satisfactory agreement with the in-plane magnetic moment data at $5 \mathrm{~K}$; see solid line in Fig. 2(b). The direction dependence shows the presence of considerable spin-orbit coupling; this means that it is too simplistic to describe the $\mathrm{Ho}^{3+}$ ions by a free ion model, but one would have to construct a spin Hamiltonian instead. Magnetic order in the Ho sublattice was not observed in magnetization measurements down to $5 \mathrm{~K}$.

\section{Magnetoelectric effect}

In this section the magnetoelectric effect is explored. Figure 3 shows the real and imaginary part of the dielectric permittivity measured along the $c$ axis of the o- $\mathrm{HoMnO}_{3}$ film. Magnetic fields were applied either in-plane or perpendicularto-plane. Both permittivity components show a maximum at a field-dependent temperature between 12 and $16 \mathrm{~K}$. The onset of the additional dielectric contribution is not very well defined for $\epsilon^{\prime}$, but is located at $22 \mathrm{~K}$ for $\epsilon^{\prime \prime}$ in agreement with Ref. 6 . There is a significant magnetoelectric effect that strongly depends on the direction of the applied magnetic field. Comparing the permittivity of $c$-axis-oriented $\mathrm{o}-\mathrm{HoMnO}_{3}$ films with that measured on bulk, ${ }^{18,29}$ one distinct difference emerges. Whereas upon cooling polycrystalline o- $\mathrm{HoMnO}_{3}$ bulk samples show a gradual onset of the permittivity at the Néel temperature of $41 \mathrm{~K}$ and a steeper rise below about $30 \mathrm{~K},{ }^{18,29}$ the permittivity of the films does not rise above the background until cooled down to about $24 \mathrm{~K}$. This suggests that measurements on polycrystals probe an average of permittivity components. Since the electric polarization has an onset below the lock-in temperature of $26 \mathrm{~K},{ }^{17,29}$ it might at first glance be related to the permittivity along the $c$ axis. Ferroelectric polarization curves measured in $c$-axisoriented films along the $c$ axis, however, are quite ellipsoidal barely resembling a proper ferroelectric loop. ${ }^{30}$ Moreover, the ferroelectricity in o- $\mathrm{HoMnO}_{3}$ is believed to be due to electronic and ionic displacements induced by the AFM $E$-type magnetic structure. ${ }^{12,14,31}$ According to that model the electric polarization is parallel to the $a$ axis such that the measured $c$-axis permittivity shown here would be due to a transverse electric susceptibility. However, ferroelectrics in general do not show transverse susceptibilities. Thus, there might be two sources for the dielectric anomalies observed here: (i) These might arise from a polarization component induced along the $c$ axis by the gradual magnetic ordering of the Ho sublattice; (ii) these are not due to ferroelectric ordering, but are induced by the magnetoresistance of $\mathrm{o}-\mathrm{HoMnO}_{3}$ via the MaxwellWagner effect. ${ }^{32,33}$

The field dependence of the dielectric permittivity is shown in Figs. 4 and 5 for the two field directions and temperatures below and above $16 \mathrm{~K}$, respectively. ${ }^{27}$ For clarity a relative dielectric permittivity is shown that is defined as $\Delta \epsilon^{\prime}(H)=$ $\epsilon^{\prime}(H)-\epsilon^{\prime}(0)$ and $\Delta \epsilon^{\prime \prime}(H)=\epsilon^{\prime \prime}(H)-\epsilon^{\prime \prime}(0)$. As for the magnetization the field direction has a significant influence on the magnetoelectric effect. For in-plane magnetic fields saturation is reached at $8 \mathrm{~T}$, at least for $\epsilon^{\prime \prime}$, whereas the dielectric permittivity is far from saturation in an $8 \mathrm{~T}$ out-of-plane field. This is very similar to the behavior of the magnetization, see Fig. 2, and indicates a strong relation between the magnetic properties of the Ho sublattice and the magnetoelectric effect. Hysteresis was observed in the dielectric permittivity up to $16 \mathrm{~K}$, but not above that temperature. This is an important observation, since field hysteresis in general indicates the presence of magnetic domains and therefore magnetic order. Since the Mn sublattice orders at higher temperatures, one might conclude that at $16 \mathrm{~K}$ either the Ho sublattice starts to order magnetically or magnetic coupling between the Ho and the Mn sublattices sets in. The maxima in the real and imaginary parts of the permittivity occur at about $16 \mathrm{~K}$, see Fig. 3, so these might be related to the onset of magnetic order in the Ho sublattice.

The angular dependence of the dielectric permittivity for field rotations in the $(110)_{o}$ and $(1 \overline{1} 0)_{o}$ planes is shown 


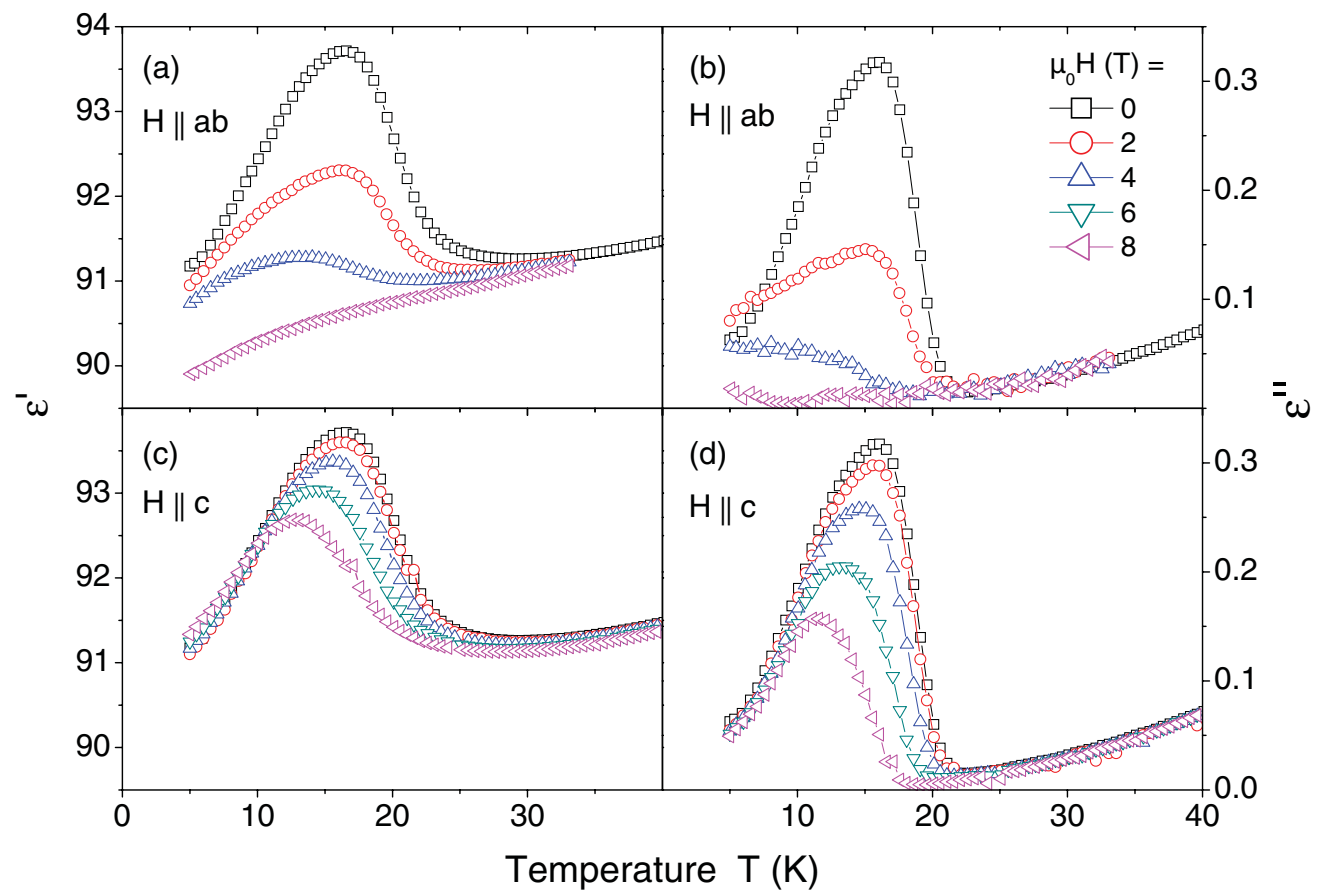

FIG. 3. (Color online) Real $\epsilon^{\prime}$ and imaginary $\epsilon^{\prime \prime}$ component of the permittivity of an o- $\mathrm{HoMnO}_{3}$ film as a function of temperature for magnetic fields applied either $[(\mathrm{a}),(\mathrm{b})]$ in-plane $\left([110]_{o}\right.$ or $\left.[1 \overline{1} 0]_{o}\right)$ or $[(\mathrm{c}),(\mathrm{d})]$ perpendicular-to-plane $\left([001]_{o}\right)$. Measurement frequency was $1 \mathrm{kHz}$.

in Fig. 6. The relative dielectric permittivity shown in this case is defined as $\Delta \epsilon^{\prime}\left(H, \theta_{F}\right)=\epsilon^{\prime}\left(H, \theta_{F}\right)-\epsilon^{\prime}\left(H, 90^{\circ}\right)$ and $\Delta \epsilon^{\prime \prime}\left(H, \theta_{F}\right)=\epsilon^{\prime \prime}\left(H, \theta_{F}\right)-\epsilon^{\prime \prime}\left(H, 90^{\circ}\right) . \theta_{F}$ denotes the angle between magnetic field and the $c$ axis. The curves have a domelike shape with the maximum at $\theta_{F}=0^{\circ}$ when the magnetic field is parallel to the $c$ axis. This is consistent with the temperature- and magnetic-field-dependent measurements that show a significantly larger magnetoelectric effect on

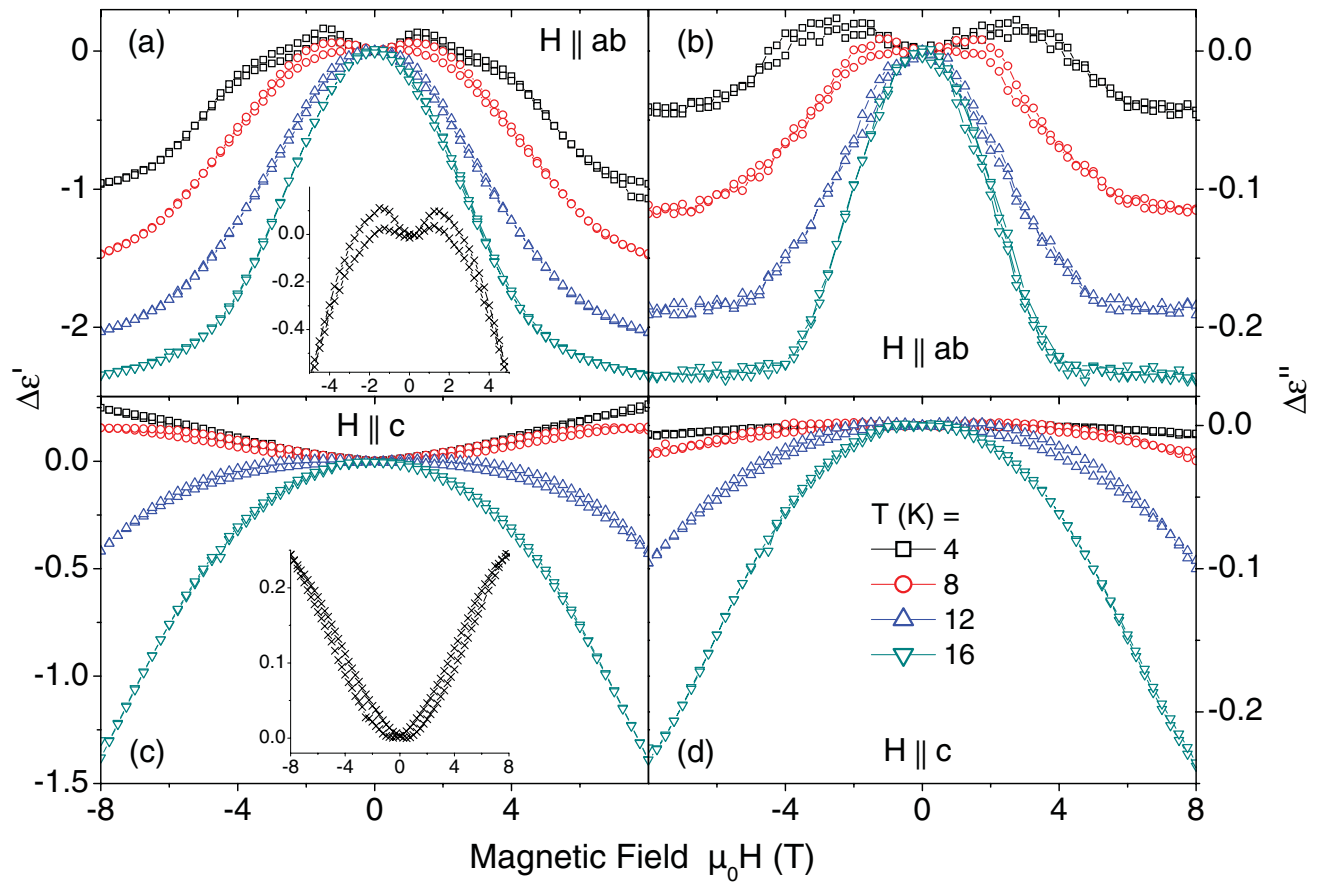

FIG. 4. (Color online) Real $\Delta \epsilon^{\prime}$ (left) and imaginary $\Delta \epsilon^{\prime \prime}$ (right) parts of the relative dielectric permittivity for various temperatures $\leqslant 16 \mathrm{~K}$ as a function of $[(\mathrm{a}),(\mathrm{b})]$ in-plane $\left([110]_{o}\right.$ or $\left.[1 \overline{1} 0]_{o}\right)$ and $[(\mathrm{c}),(\mathrm{d})]$ perpendicular-to-plane $\left([001]_{o}\right)$ magnetic fields. The curves were shifted such that the permittivity is zero in zero magnetic field. The insets in (a) and (c) show the $6 \mathrm{~K}$ data on an expanded scale to highlight the magnetic hysteresis. Variables and units of the inset axes are the same as for the main-panel axes. 


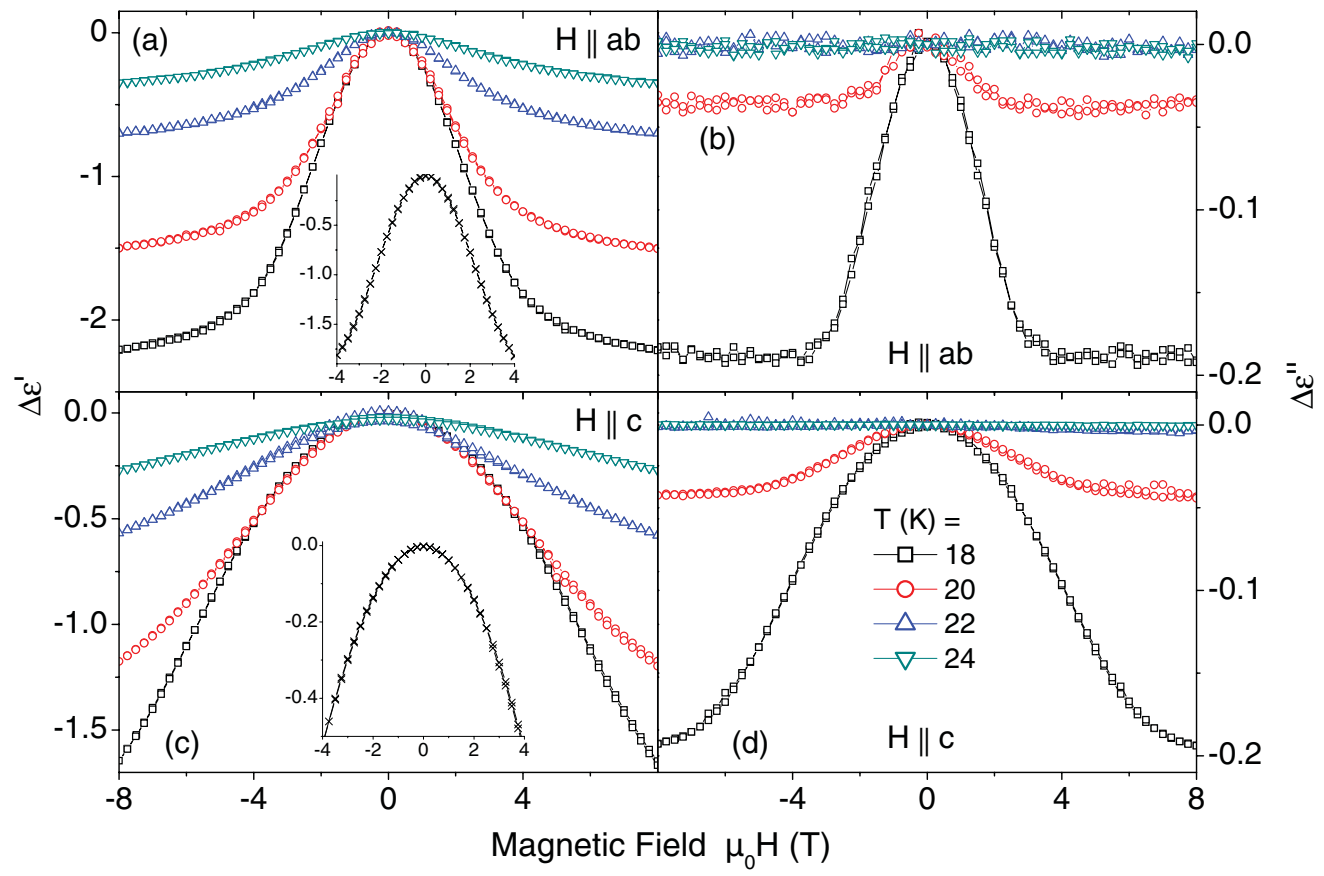

FIG. 5. (Color online) Real $\Delta \epsilon^{\prime}$ (left) and imaginary $\Delta \epsilon^{\prime \prime}$ (right) parts of the relative dielectric permittivity for various temperatures $>16 \mathrm{~K}$ as a function of $[(\mathrm{a}),(\mathrm{b})]$ in-plane $\left([110]_{o}\right.$ or $\left.[1 \overline{1} 0]_{o}\right)$ and $[(\mathrm{c}),(\mathrm{d})]$ perpendicular-to-plane $\left([001]_{o}\right)$ magnetic fields. The curves were shifted such that the permittivity is zero in zero magnetic field. The insets in (a) and (c) show the $18 \mathrm{~K}$ data on an expanded scale to highlight the absence of magnetic hysteresis. Variables and units of the inset axes are the same as for the main-panel axes.

the dielectric permittivity for in-plane than for out-of-plane fields. The choice of the rotation axes does not allow for a discrimination between the orthorhombic $a$ and $b$ axes. However, since the curves for the two rotation planes are identical within experimental error, one can directly conclude that the $c$ axis is along the surface normal in agreement with the $\mathrm{x}$-ray results. As deduced from symmetry considerations in the Appendix one would expect that the angular dependence

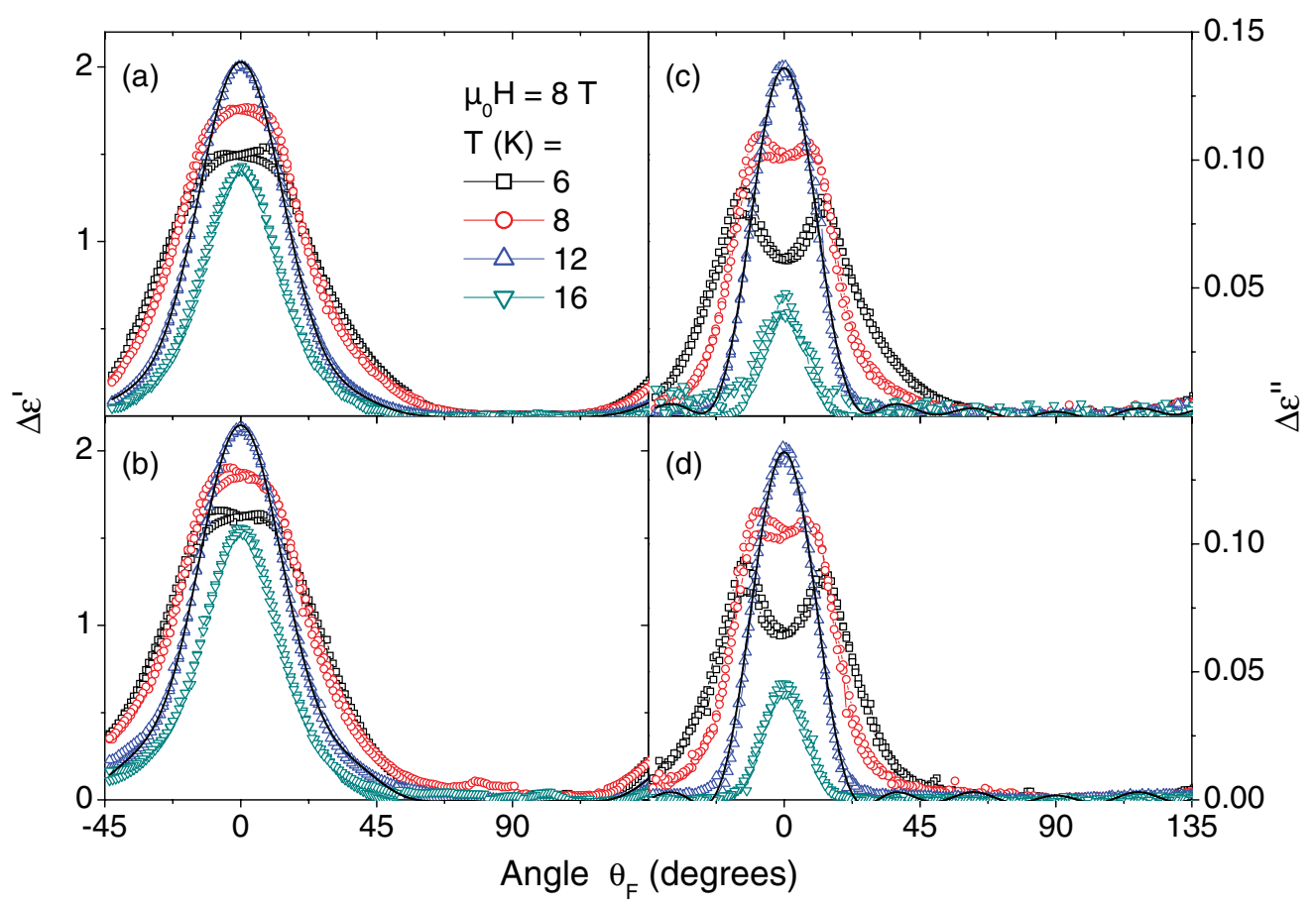

FIG. 6. (Color online) Angular dependence of the [(a), (b)] real and [(c), (d)] imaginary part of the dielectric permittivity for field rotations in the [(a), (c)] (110) $)_{o}$ and [(b), (d)] (11) $)_{o}$ planes. The solid lines through the $12 \mathrm{~K}$ data are fits of a model based on symmetry considerations to the data. The permittivity curves were shifted such that $\Delta \hat{\epsilon}$ was zero in the in-plane direction $\left(90^{\circ}\right)$. 


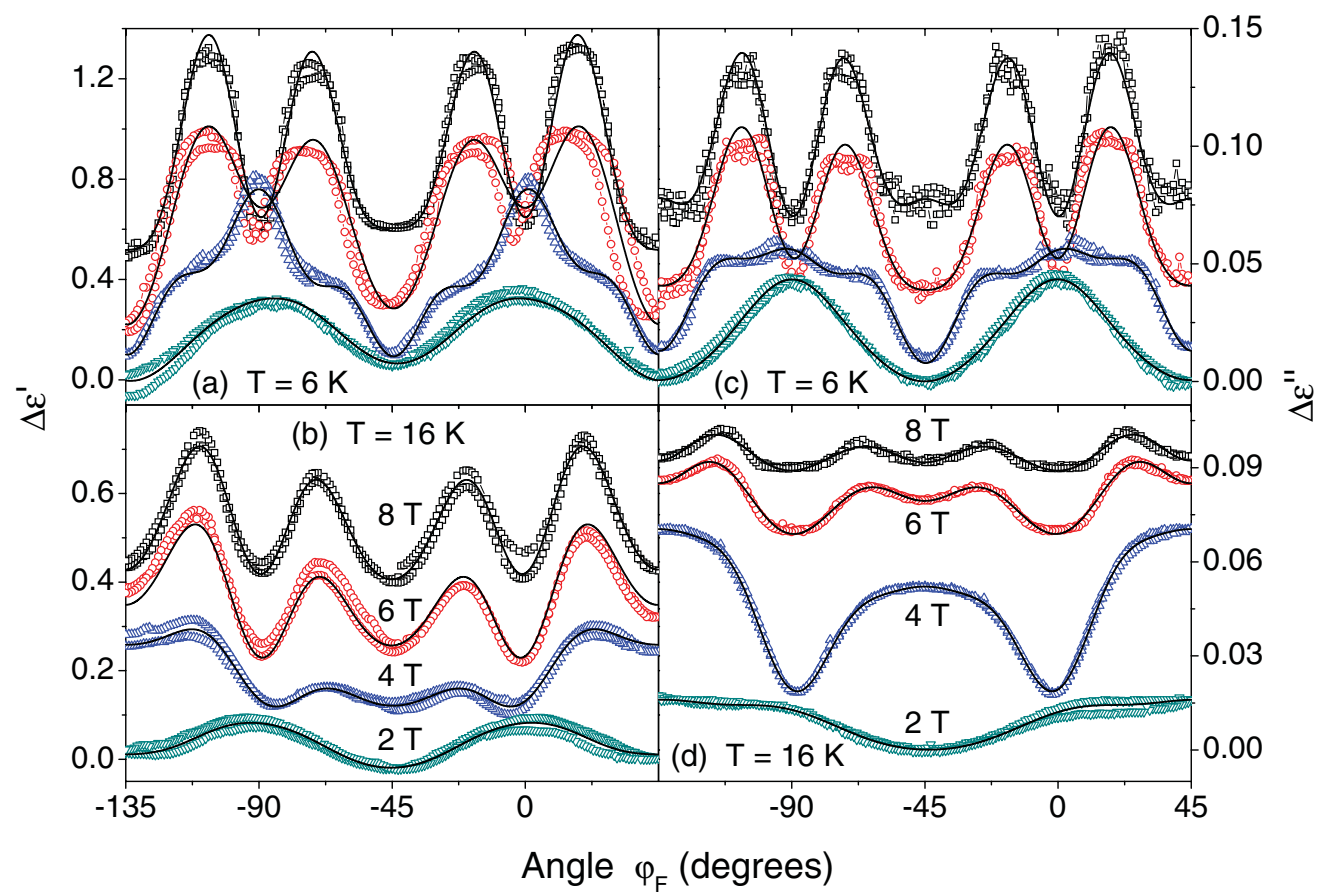

FIG. 7. (Color online) Angular dependence of the [(a), (b)] real and [(c), (d)] imaginary part of the dielectric permittivity for field rotations in the $(001)_{o}$ plane at $[(a),(c)] 6 \mathrm{~K}$ and $[(b),(d)] 16 \mathrm{~K}$. The solid lines are fits of a model based on symmetry considerations to the data. For clarity some curves were upshifted.

follows the relation

$$
\hat{\epsilon}=\epsilon^{\prime}-i \epsilon^{\prime \prime}=\sum_{n=0}^{\infty}\left(\epsilon_{2 n}^{\prime}-i \epsilon_{2 n}^{\prime \prime}\right) \cos (2 n \theta),
$$

where $\theta$ denotes the angle between the magnetization and the $c$ axis and the $\hat{\epsilon}_{2 n}=\epsilon_{2 n}^{\prime}-i \epsilon_{2 n}^{\prime \prime}$ are expansion coefficients. The magnetic-field-dependent permittivity curves shown in Figs. 4 and 5 indicate that the permittivity is close to saturation in a field of $8 \mathrm{~T}$ such that $\theta \simeq \theta_{F}$ at least in the reversible regime.

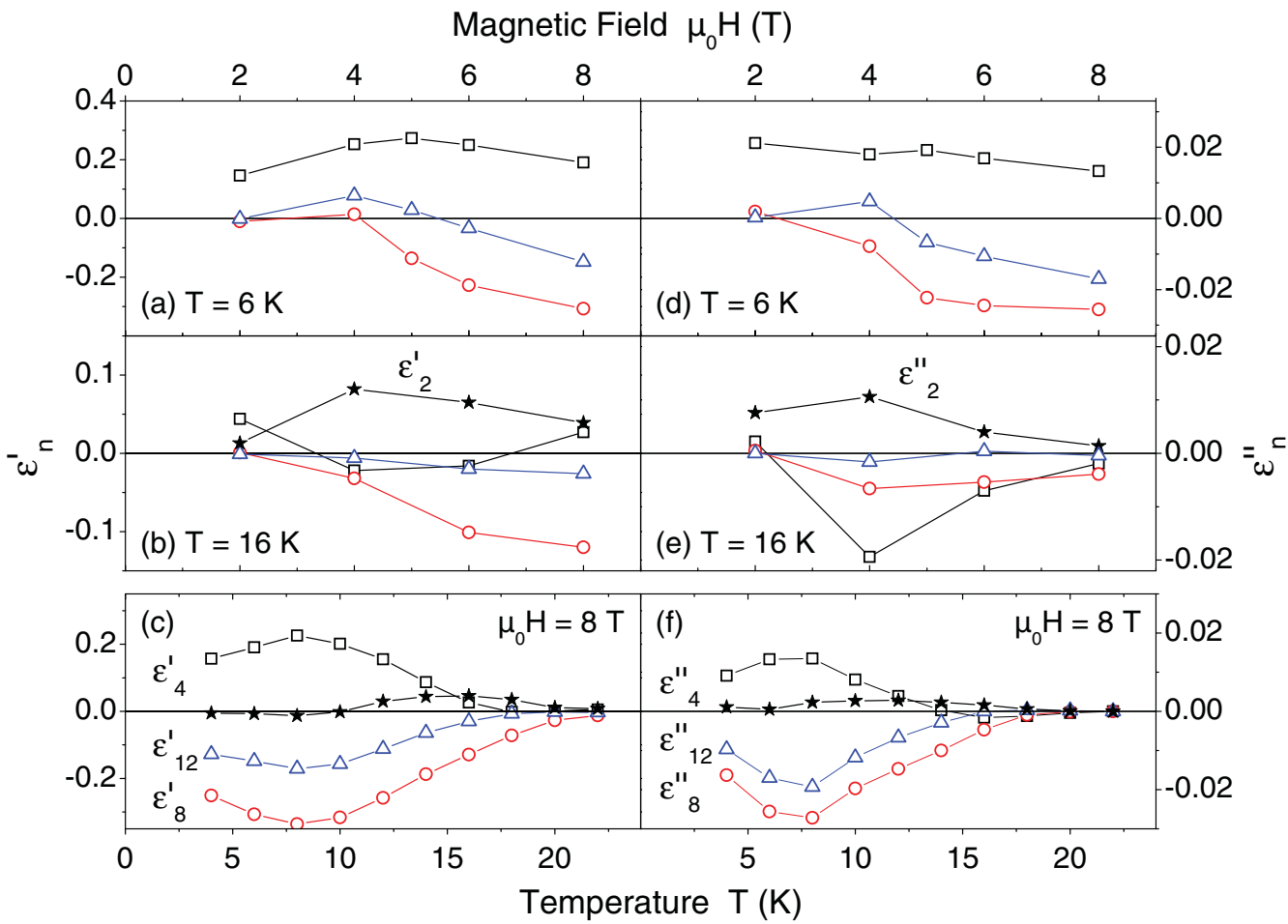

FIG. 8. (Color online) Coefficients of Eq. (2) as determined from the in-plane angular rotation measurements. 
Equation (1) yields a good fit to the data at $12 \mathrm{~K}$ and higher temperatures, see Fig. 6, but cannot properly fit the data at lower temperatures, since these show some hysteresis. The latter is consistent with the hysteresis observed in the field dependence of the dielectric permittivity, see Figs. 4 and 5, again indicating magnetic order in the Ho sublattice.

The corresponding angular dependence of the dielectric permittivity for field rotation in the $(001)_{o}$ plane is shown in Fig. 7. The relative dielectric permittivity shown in this case is defined as $\Delta \epsilon^{\prime}\left(H, \varphi_{F}\right)=\epsilon^{\prime}\left(H, \varphi_{F}\right)-\epsilon^{\prime}\left(H,-45^{\circ}\right)$ and $\Delta \epsilon^{\prime \prime}\left(H, \varphi_{F}\right)=\epsilon^{\prime \prime}\left(H, \varphi_{F}\right)-\epsilon^{\prime \prime}\left(H,-45^{\circ}\right) . \varphi_{F}$ denotes the angle between magnetic field and an edge of the substrate; i.e., $\varphi_{F}=0^{\circ}$ corresponds to either the $[110]_{o}$ or $[1 \overline{1} 0]_{o}$ direction. Both at 6 and $16 \mathrm{~K}$ the permittivity curves show a crossover from a fourfold rotation pattern at low fields to a higher order rotation pattern at high fields. From symmetry considerations one would expect that the permittivity follows, see Appendix,

$$
\begin{aligned}
\hat{\epsilon}= & \epsilon^{\prime}-i \epsilon^{\prime \prime}=\sum_{n=0}^{\infty} \hat{\epsilon}_{4 n} \cos (4 n \varphi) \\
& +\sum_{n=1}^{\infty} \hat{\epsilon}_{(4 n-2)} \sin [(4 n-2) \varphi],
\end{aligned}
$$

with complex expansion coefficients $\hat{\epsilon}_{2 n}=\epsilon_{2 n}^{\prime}-i \epsilon_{2 n}^{\prime \prime}$ that are not identical in magnitude to the coefficients in Eq. (1). Note that this expression contains cosine terms of order $4 n$ but sine terms of order $4 n-2$. Since the curves do not show rotational hysteresis within experimental error, Eq. (2) was fitted to the data under the assumption $\varphi=\varphi_{F}$, i.e., that the magnetization vector follows the magnetic field vector. The data can be convincingly analyzed with this expression using cosine terms up to 12 th order and a sine term of only second order. Note that the coefficient $\hat{\epsilon}_{2}$ of the sine term is negligible below $12 \mathrm{~K}$. The corresponding coefficients, $\hat{\epsilon}_{4}, \hat{\epsilon}_{8}, \hat{\epsilon}_{12}$, and $\hat{\epsilon}_{2}$, are shown in Fig. 8 as a function of magnetic field and temperature. This analysis reveals a striking similarity in the magnetic field and temperature dependence of the real and imaginary coefficients of the complex permittivity; i.e., the angular dependence of the permittivity follows the conductance and vice versa.

\section{Discussion}

For a further discussion the main findings from the measurements of the magnetoelectric effect are summarized:

(1) An anomaly in the complex permittivity was observed with a maximum around $16 \mathrm{~K}$ in both permittivity components.

(2) Below $16 \mathrm{~K}$ hysteresis appeared in the magnetic field dependence of the permittivity.

(3) The form of the magnetic-field-dependent permittivity curves is reminiscent of a $M^{2}$ dependence.

(4) There is a large anisotropy of the complex permittivity for in- and out-of-plane magnetic field orientations.

(5) The in-plane permittivity shows a crossover from a fourfold to a more complex rotation pattern on increasing the magnetic field strength.

(6) Both real and imaginary parts of the complex permittivity show strikingly similar magnetic field and angle dependence, albeit with the absolute effects being smaller in the imaginary component by a factor of about ten.
From (3) and the fact that the magnetization predominantly reflects the properties of $\mathrm{Ho}^{3+}$ ions one might conclude that the Ho sublattice strongly affects the complex permittivity. Since ordering of Ho moments was not observed in magnetization measurements, the magnetic hysteresis of the permittivity (2) indicates a coupling of Ho and Mn moments. Observation (5) indicates that Ho moments also dominate the anisotropy of the magnetoelectric effect, since the crossover in rotational anisotropy occurs when the Ho sublattice starts to saturate.

Observation (6) might highlight the underlying mechanism for the magnetoelectric effect. In a ferroelectric material one might expect the electric polarization and the conductance to be dominated by different physical processes. It is therefore striking that both quantities show a very similar temperature, magnetic field, and angle dependence. This might be naturally explained by the Maxwell-Wagner effect ${ }^{32}$ that leads to magnetoelectric behavior induced solely by magnetoresistance. ${ }^{33}$ The o- $\mathrm{HoMnO}_{3}$ sample under study might be composed of layers with different electric properties, e.g., a strained layer close to the o- $\mathrm{HoMnO}_{3}-\mathrm{Nb}: \mathrm{SrTiO}_{3}$ interface and a strainrelaxed layer close to the $\mathrm{Au}$ contact; moreover, Schottky barriers at the contacts might play a role. This would lead to an interfacial polarization and a complex permittivity depending on the conductances and the relaxation times of both layers. ${ }^{33,34}$ Although the absolute change in the permittivity is larger for the real than for the loss component, the relative change in the loss component is much larger than that in the real one, e.g., $30 \%$ in $\epsilon^{\prime \prime}$ vs $0.8 \%$ in $\epsilon^{\prime}$ at $6 \mathrm{~K}$ and $8 \mathrm{~T}$. It appears therefore that a rather large modulation of the conductance leads to a small modulation of the effective real component of the permittivity via interfacial polarization effects. The frequency-dependent data available for this sample ${ }^{19}$ show a slightly frequency-dependent permittivity and magnetoelectric effect and are consistent with this scenario. Besides, the interpretation within the Maxwell-Wagner effect accounts for the observation that the measured magnetoelectric effect is perpendicular to the electric polarization.

\section{CONCLUSIONS}

In this work the structural, magnetic, and magnetoelectric properties of o- $\mathrm{HoMnO}_{3}$ films grown on $0.5 \% \mathrm{Nb}$-doped $\mathrm{SrTiO}_{3}$ (001) substrates were studied. The films were found to have orthorhombic symmetry with a uniform crystallographic orientation with the $c$ axis along the film normal and the $a$ and $b$ axes in the film plane; this result excludes twinning in the films. The magnetization of the films is dominated by the paramagnetism of the $\mathrm{Ho}^{3+}$ ions; an antiferromagnetic transition of the Mn sublattice was not observed. The paramagnetic magnetization is strongly anisotropic with respect to the inand out-of-plane magnetic field direction. In the real and loss part of the permittivity an anomaly below about $24 \mathrm{~K}$ with a maximum at about $16 \mathrm{~K}$ was observed. This is strongly magnetic field dependent and can be fully suppressed by an $8 \mathrm{~T}$ in-plane field. Measurements of the in-plane rotational anisotropy of the permittivity showed an intricate angular dependence with a field-dependent transition from a fourfold rotation pattern at low fields to a rotation pattern containing up to 12 th order terms at higher magnetic fields. Since the magnetic field dependence of the permittivity corresponds 
to that of the paramagnetic magnetization component, the rotational anisotropy of the permittivity arises from spin rotations in the Ho sublattice. Picozzi et al. ${ }^{12}$ studied the polarization reversal by $\mathrm{Mn}$ spin rotation and found a $\cos \varphi$ dependence of the polarization that cannot account for the complicated rotational anisotropy observed here. Therefore our results call for the study of the interaction between the Ho sublattice magnetization and the electric polarization. The magnetoelectric effect probably arises from magnetoresistance within the Maxwell-Wagner effect.

\section{ACKNOWLEDGMENTS}

This work was supported by the German Science Foundation (DFG) within the Collaborative Research Center SFB 762 "Functionality of Oxide Interfaces."

\section{APPENDIX}

A possible starting point for the description of the angulardependent magnetic and electric properties would be the expansion of the Gibbs free energy in terms of the electric $\vec{E}$ and magnetic $\vec{H}$ fields. ${ }^{4}$ Within this formalism an electric polarization induced by the rotation of a magnetic field and having an angle dependence $\cos (2 n \varphi)$ could be understood as a rising from the expansion to the $2 n$th order in $\vec{H}$. It is unlikely, however, that terms of the type $\cos (12 \varphi)$ which were experimentally observed are due to the magnetically induced polarization, since magnetoelectrical susceptibilities of 12 th order are expected to be much too small to explain the effect. Therefore the angular dependence of the electric susceptibility observed here might arise from the dependence of the permittivity and/or magnetoconductance on the direction of the magnetization $\vec{M}_{S}=M_{S}(\sin \theta \cos \varphi, \sin \theta \sin \varphi, \cos \theta)$. Here $\theta$ and $\varphi$ are the angles of a spherical coordinate system defined with respect to the cubic $[001]_{c}$ and $[100]_{c}$ substrate directions, as indicated in Fig. 9.

Here the complex permittivity $\hat{\epsilon}=C_{0}^{-1}(C-i G / \omega)$ was measured that is directly related to the conductance along the orthorhombic $c$ axis. The angular dependence of $\hat{\epsilon}$ is unknown in detail, but is restricted by the crystalline symmetry

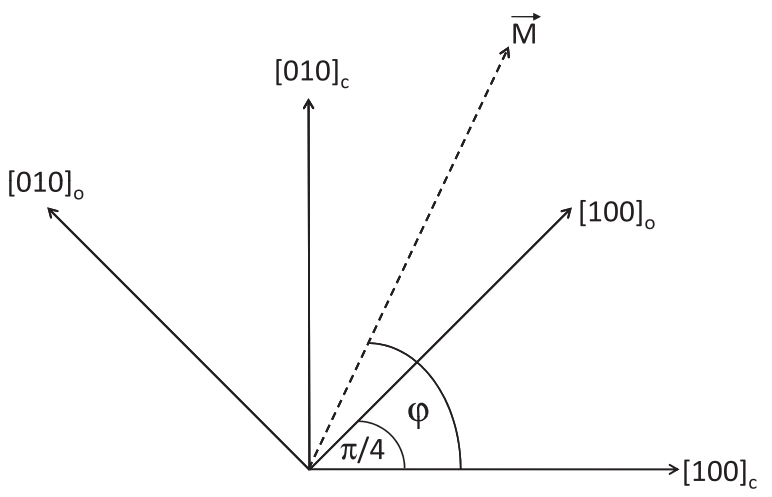

FIG. 9. Configuration of the in-plane magnetic field rotations. The sketch shows the magnetization vector with respect to the cubic basis vectors of the $\mathrm{SrTiO}_{3}$ substrate and the orthorhombic basis vectors of the o- $\mathrm{HoMnO}_{3}$ film. of the o- $\mathrm{HoMnO}_{3}$ film. A related problem was treated by Döring and Simon ${ }^{35,36}$ in the case of the dependence of the magnetostriction and anisotropic magnetoresistance on the direction of the saturation magnetization. Here we follow the treatment outlined in Refs. 35,36 to derive the angular dependence of the electric susceptibility.

Let $\alpha_{i}$ denote the direction cosines of the magnetization $\vec{M}_{S}=M_{S}\left(\alpha_{1}, \alpha_{2}, \alpha_{3}\right)$ with respect to the orthorhombic axes of the o- $\mathrm{HoMnO}_{3}$ film. In general $\hat{\epsilon}$ can be expressed by

$$
\hat{\epsilon}=\sum_{i j=1}^{3} A_{i j} \beta_{i} \beta_{j}
$$

where $\beta_{i}$ denote the direction cosines of the driving force, i.e., the electric field or the current density, with respect to the basis vectors. The matrix elements $A_{i j}$ depend on the direction cosines $\alpha_{i}$ with the functional dependence being restricted by the crystallographic symmetry.

For an electric field along the $c$ axis of the orthorhombic lattice one has $\beta_{1}=\beta_{2}=0, \beta_{3}=1$, and in orthorhombic $D_{2 h}$ symmetry

$$
\hat{\epsilon}=A_{33}=K\left(\alpha_{1}^{2}, \alpha_{2}^{2}\right),
$$

where $K$ denotes an unknown function of the arguments $\alpha_{1}^{2}$ and $\alpha_{2}^{2} .{ }^{35} K$ can be expanded in the direction cosines and can through these be expressed by the angle between the magnetization and a given axis.

(1) Rotation in the $(001)_{o}$ plane

For this case Fig. 9 shows the magnetization direction with respect to the cubic axes of the $\mathrm{SrTiO}_{3}$ substrate and the orthorhombic axes of the o- $\mathrm{HoMnO}_{3}$ film. Experimentally the angle $\varphi$ was defined with respect to a cubic [100] axis. Since the orthorhombic axes are rotated with respect to the cubic ones by $45^{\circ}$, the direction cosines are given by $\alpha_{1}=(\sin \varphi+\cos \varphi) / \sqrt{2}, \alpha_{2}=(\sin \varphi-\cos \varphi) / \sqrt{2}$, and $\alpha_{3}=0 . \varphi$ denotes the angle between magnetization vector and $[100]_{c}$. This yields an expansion

$$
\hat{\epsilon}=\hat{\epsilon}_{0}+\sum_{n=1}^{\infty} \hat{\epsilon}_{4 n-2} \sin [(4 n-2) \varphi]+\sum_{n=1}^{\infty} \hat{\epsilon}_{4 n} \cos (4 n \varphi) .
$$

\section{(2) Rotation in the $(1 \overline{1} 0)_{o}$ plane}

In this case the direction cosines are $\alpha_{1}=\sin \theta / \sqrt{2}, \alpha_{2}=$ $\sin \theta / \sqrt{2}$, and $\alpha_{3}=\cos \theta$, where $\theta$ denotes the angle between the magnetization vector and $[001]_{o}$, the substrate normal. This yields an expansion

$$
\hat{\epsilon}=\hat{\epsilon}_{0}+\sum_{n=1}^{\infty} \hat{\epsilon}_{2 n} \cos (2 n \theta) .
$$

\section{(3) Rotation in the (110)o plane}

This situation is equivalent to the previous situation, since the $(110)_{o}$ and $(1 \overline{1} 0)_{o}$ planes are equivalent for orthorhombic symmetry. A rotation in the $(100)_{o}$ or $(010)_{o}$ planes would again yield the same form of the expansion, Eq. (A4), albeit with different coefficients. 
${ }^{1}$ T. Kimura, T. Goto, H. Shintani, K. Ishizaka, T. Arima, and Y. Tokura, Nature (London) 426, 55 (2003).

${ }^{2}$ N. Hur, S. Park, P. A. Sharma, J. S. Ahn, S. Guha, and S.-W. Cheong, Nature (London) 429, 392 (2004).

${ }^{3}$ T. Goto, T. Kimura, G. Lawes, A. P. Ramirez, and Y. Tokura, Phys. Rev. Lett. 92, 257201 (2004).

${ }^{4}$ M. Fiebig, J. Phys. D: Appl. Phys. 38, R123 (2005).

${ }^{5}$ T. H. Lin, C. C. Hsieh, H. C. Shih, C. W. Luo, T. M. Uen, K. H. Wu, J. Y. Juang, J.-Y. Lin, C.-H. Hsu, and S. J. Liu, Appl. Phys. Lett. 92, 132503 (2008).

${ }^{6}$ T. H. Lin, H. C. Shih, C. C. Hsieh, C. W. Luo, J.-Y. Lin, J. L. Her, H. D. Yang, C.-H. Hsu, K. H. Wu, T. M. Uen, and J. Y. Juang, J. Phys. Condens. Matter 21, 026013 (2009).

${ }^{7}$ T. H. Lin, C. C. Hsieh, H. C. Shih, C. W. Luo, T. M. Uen, K. H. Wu, J.-Y. Lin, C.-H. Hsu, and J. Y. Juang, J. Phys.: Conf. Ser. 150, 042114 (2009).

${ }^{8}$ T. C. Han and J. G. Lin, Appl. Phys. Lett. 94, 082502 (2009).

${ }^{9}$ T.-C. Han, J. G. Lin, C.-T. Wu, M.-W. Chu, and C.-H. Chen, Jpn. J. Appl. Phys. 49, 041501 (2010).

${ }^{10}$ H. W. Brinks, J. Rodríguez-Carvajal, H. Fjellvåg, A. Kjekshus, and B. C. Hauback, Phys. Rev. B 63, 094411 (2001).

${ }^{11}$ A. Muñoz, M. T. Casaís, J. A. Alonso, M. J. Martínez-Lope, J. L. Martínez, and M. T. Fernández-Díaz, Inorg. Chem. 40, 1020 (2001).

${ }^{12}$ S. Picozzi, K. Yamauchi, B. Sanyal, I. A. Sergienko, and E. Dagotto, Phys. Rev. Lett. 99, 227201 (2007).

${ }^{13}$ S. Picozzi, K. Yamauchi, G. Bihlmayer, and S. Blügel, Phys. Rev. B 74, 094402 (2006).

${ }^{14}$ K. Yamauchi, F. Freimuth, S. Blügel, and S. Picozzi, Phys. Rev. B 78, 014403 (2008).

${ }^{15}$ S. Picozzi and C. Ederer, J. Phys. Condens. Matter 21, 303201 (2009).

${ }^{16}$ V. E. Wood, A. E. Austin, E. W. Collings, and K. C. Brog, J. Phys. Chem. Solids 34, 859 (1973).

${ }^{17}$ B. Lorenz, Y. Q. Wang, and C. W. Chu, Phys. Rev. B 76, 104405 (2007).

${ }^{18}$ B. Lorenz, Y. Q. Wang, Y. Y. Sun, and C. W. Chu, Phys. Rev. B 70, 212412 (2004).
${ }^{19}$ R. Wunderlich, C. Chiliotte, G. Bridoux, T. Maity, Ö. Kocabiyik, A. Setzer, M. Ziese, and P. Esquinazi, J. Magn. Magn. Mater. 324, 460 (2012).

${ }^{20}$ Q. Gan, R. A. Rao, C. B. Eom, L. Wu, and F. Tsui, J. Appl. Phys. 85, 5297 (1999).

${ }^{21}$ S. Venkatesan, C. Daumont, B. J. Kooi, B. Noheda, and J. T. M. De Hosson, Phys. Rev. B 80, 214111 (2009).

${ }^{22}$ R. A. Rao, D. Lavric, T. K. Nath, C. B. Eom, L. Wu, and F. Tsui, J. Appl. Phys. 85, 4794 (1999).

${ }^{23}$ M. Ziese, H. C. Semmelhack, K. H. Han, S. P. Sena, and H. J. Blythe, J. Appl. Phys. 91, 9930 (2002).

${ }^{24}$ L. S.-J. Peng, X. X. Xi, B. H. Moeckly, and S. P. Alpay, Appl. Phys. Lett. 83, 4592 (2003).

${ }^{25} \mathrm{X}$. Marti, V. Skumryev, C. Ferrater, M. V. García-Cuenca, M. Varela, F. Sánchez, and J. Fontcuberta, Appl. Phys. Lett. 96, 222505 (2010)

${ }^{26} \mathrm{P}$. Boulanger and M. Hayes, J. Elasticity 50, 87 (1998).

${ }^{27}$ S.-H. Lee, M.-H. Jung, C.-H. Yang, T. Y. Koo, and Y. H. Jeong, J. Phys.: Conf. Ser. 200, 012103 (2010).

${ }^{28}$ N. Jehanathan, O. Lebedev, I. Gélard, C. Dubourdieu, and G. Van Tendeloo, Nanotechnology 21, 075705 (2010).

${ }^{29}$ S. M. Feng, Y. S. Chai, J. L. Zhu, N. Manivannan, Y. S. Oh, L. J. Wang, Y. S. Yang, C. Q. Jin, and K. H. Kim, New J. Phys. 12, 073006 (2010)

${ }^{30}$ T. H. Lin, C. C. Hsieh, C. W. Luo, J.-Y. Lin, C. P. Sun, H. D. Yang, C.-H. Hsu, Y. H. Chu, K. H. Wu, T. M. Uen, and J. Y. Juang, J. Appl. Phys. 106, 103923 (2009).

${ }^{31}$ I. A. Sergienko, C. Şen, and E. Dagotto, Phys. Rev. Lett. 97, 227204 (2006).

${ }^{32}$ A. R. von Hippel, Dielectrics and Waves (John Wiley \& Sons, New York, 1954), pp. 228-234.

${ }^{33}$ G. Catalan, Appl. Phys. Lett. 88, 102902 (2006).

${ }^{34}$ R. Schmidt, W. Eerenstein, T. Winiecki, F. D. Morrison, and P. A. Midgley, Phys. Rev. B 75, 245111 (2007).

${ }^{35}$ W. Döring and G. Simon, Ann. Phys. 460, 373 (1960).

${ }^{36}$ W. Döring and G. Simon, Ann. Phys. 463, 144 (1961). 